\title{
Positive and negative functions of the SAGA complex mediated through interaction of Spt8 with TBP and the $\mathrm{N}$-terminal domain of TFIIA
}

\author{
Linda Warfield, ${ }^{1}$ Jeffrey A. Ranish, ${ }^{2}$ and Steven Hahn ${ }^{1,3}$ \\ ${ }^{1}$ Fred Hutchinson Cancer Research Center, and Howard Hughes Medical Institute, Seattle, Washington 98109, USA; \\ and ${ }^{2}$ Institute for Systems Biology, Seattle, Washington 98103, USA
}

\begin{abstract}
A surface that is required for rapid formation of preinitiation complexes (PICs) was identified on the N-terminal domain (NTD) of the RNA Pol II general transcription factor TFIIA. Site-specific photocross-linkers and tethered protein cleavage reagents positioned on the NTD of TFIIA and assembled in PICs identified the SAGA subunit Spt8 and the TFIID subunit Taf4 as located near this surface. In agreement with these findings, mutations in Spt8 and the TFIIA NTD interact genetically. Using purified proteins, it was found that TFIIA and Spt8 do not stably bind to each other, but rather both compete for binding to TBP. Consistent with this competition, Spt8 inhibits the binding of SAGA to PICs in the absence of activator. In the presence of activator, Spt8 enhances transcription in vitro, and the positive function of the TFIIA NTD is largely mediated through Spt8. Our results suggest a mechanism for the previously observed positive and negative effects of Spt8 on transcription observed in vivo.
\end{abstract}

[Keywords: Transcription; SAGA; TFIIA; coactivator; repression]

Supplemental material is available at http://www.genesdev.org.

Received February 6, 2004; revised version accepted March 22, 2004.

Binding of early-acting gene-specific factors to gene regulatory sequences and recruitment of the chromatin remodeling machinery initiates a cascade of events in which transcription coactivators and the general transcription machinery are recruited to promoters, forming a preinitiation complex (PIC; Kuras and Struhl 1999; Li et al. 1999; Agalioti et al. 2000; Cosma 2002). The recruitment of TBP, a DNA sequence-specific binding component of PICs, is regulated both positively and negatively (Hampsey 1998). In many cases, activators increase the binding of TBP to promoters in direct proportion to their activity in transcription activation (Kuras and Struhl 1999; Li et al. 1999). In contrast, factors such as Mot1 or the Taf1 N-terminal domain (TAND) inhibit the DNA binding activity of TBP (Auble et al. 1994; Kokubo et al. 1998; Cang et al. 1999). The general factor TFIIA interacts directly with TBP and stabilizes TBPDNA interactions (Weideman et al. 1997; Hampsey 1998; Liu et al. 1999). TFIIA also stimulates and stabilizes the binding of TFIID to DNA as part of an activator-TFIID-TFIIA-DNA complex, a rate-limiting inter-

${ }^{3}$ Corresponding author.

E-MAIL shahn@fhcrc.org; FAX (206) 667-6497.

Article and publication are at http://www.genesdev.org/cgi/doi/10.1101/ $\operatorname{gad} .1192204$ mediate in the transcription of certain promoters (Wang et al. 1992; Lieberman and Berk 1994; Chi et al. 1995). Finally, TFIIA can compete with negative factors such as NC2, Mot1, and the Taf1 TAND domain for binding to TBP (Hampsey 1998; Kokubo et al. 1998; Ozer et al. 1998b; Cang et al. 1999). TFIIA has also been found to interact with at least two Tafs and some transcription activators, using two-hybrid and affinity chromatography analysis (Yokomori et al. 1993; Ozer et al. 1994; Kobayashi et al. 1998; Kraemer et al. 2001).

TFIIA is unique in that it is the only Pol II general factor that is not absolutely required for transcription under standard conditions. In vitro, TFIIA stimulates both basal and activated transcription from 2- to 10-fold, but generally only when the TFIID form of TBP is used (Orphanides et al. 1996; Hampsey 1998). Several approaches have been taken to determine which genes require TFIIA for expression in vivo. These include TFIIA subunit depletion, mutation of TFIIA subunits to impair interaction with TBP, or mutation of TBP to inhibit interaction with TFIIA (Kang et al. 1995; Ozer et al. 1998a; Liu et al. 1999; Stargell et al. 2000). These studies generally agree that TFIIA is important for transcription of a specific subset of genes, although there is not yet agreement on the exact subset of genes requiring TFIIA for normal expression. 
The SAGA complex is a cofactor required for normal expression of some genes in yeast (Sterner et al. 1999; Lee et al. 2000). On gene activation in vivo, SAGA can be recruited to promoters independently of the general transcription factors (Bhaumik and Green 2001; Larschan and Winston 2001). The SAGA complex contains a core comprising Ada and Spt subunits, a subset of Tafs, acetyltransferase and ubiquitin protease activities, the essential factor Tra1, and two factors related to TBP function, Spt3 and Spt8 (Grant et al. 1998; Sterner et al. 1999; Pray-Grant et al. 2002; Sanders et al. 2002). SAGA is closely related to the SLIK/SALSA complex, which lacks Spt8 but which contains at least one unique subunit, Rtg2 (Pray-Grant et al. 2002; Sterner et al. 2002; Wu and Winston 2002). It is not yet known how redundantly these two related complexes function in vivo. Only a small percentage of promoters seem to be exclusively SAGA- or TFIID-dependent in vivo (Lee et al. 2000). In general, the requirement of a particular promoter for SAGA or TFIID is determined by the core promoter sequence, and the particular activator determines whether SAGA or TFIID is recruited to a promoter (Shen and Green 1997; Cheng et al. 2002; Mencia et al. 2002). It seems that many yeast promoters can use either SAGA or TFIID, because a double mutation of SAGA and TFIID subunits or a mutation of a shared TFIID/SAGA subunit affects expression from many more yeast genes than does a single mutation of either TFIID or SAGA specific subunits (Lee et al. 2000).

At SAGA-dependent promoters, SAGA is required for recruitment of the transcription machinery (Dudley et al. 1999; Bhaumik and Green 2001; Larschan and Winston 2001). Although recruitment of SAGA to promoters is independent of $\mathrm{Spt} 3$ and $\mathrm{Spt} 8$, these subunits are required for general factor recruitment at most SAGA-dependent promoters tested (Bhaumik and Green 2001, 2002; Larschan and Winston 2001). Taken together, these observations suggest that SAGA is a coactivator that links some activators to the general transcription machinery. The mechanism whereby SAGA interacts with the general transcription machinery is not well understood. It has been proposed that the Spt3 subunit interacts directly with TBP (Bhaumik and Green 2001; Larschan and Winston 2001). Mutations on opposite sides of the TBP surface have been found to suppress an Spt3 mutation, and a mutation in Spt3 was found to suppress TBP mutations (Eisenmann et al. 1992). Spt3 was found to coimmune precipitate with TBP, but only when overexpressed. This may have been due to nonspecific interaction because Spt8, another SAGA subunit, did not coimmune precipitate with TBP (Eisenmann et al. 1992, 1994). Protein-protein interaction studies and two-hybrid analysis have not shown any direct interaction between Spt3 and TBP (Eisenmann et al. 1994; Madison and Winston 1997). In contrast, intact SAGA was found to interact with TBP and this interaction required the Spt8 and Ada3 subunits but was independent of Spt3 (Saleh et al. 1997; Sterner et al. 1999; Sanders et al. 2002).

In this work, we examine the function of the TFIIA
$\mathrm{N}$-terminal domain (NTD), an essential region of TFIIA located opposite of TBP and DNA in the TFIIA-TBPDNA complex structure (Geiger et al. 1996; Tan et al. 1996). Using site-specific protein cross-linking and a tethered protein cleavage reagent positioned near the functional surface of the NTD, we determine the identity of polypeptides located near this surface in PICs and show surprising positive and negative interactions among the SAGA subunit Spt8, TBP, and the NTD of TFIIA.

\section{Results}

A surface on the TFIIA N-terminal domain required for rapid PIC formation

Previously, alanine-scanning mutagenesis of the two TFIIA subunits, Toa1 and Toa2, identified a double alanine substitution of Toa2 residues D21 and D24 affecting the function of TFIIA in vivo (Kang et al. 1995). This double mutation, located on the surface of the four-helix bundle of the TFIIA NTD, was temperature sensitive in vivo and defective for both basal and activated transcription in vitro. As expected from the structure of the TFIIA-TBP-DNA complex (Geiger et al. 1996; Tan et al. 1996), these mutations did not affect interaction of TFIIA with TBP, formation of the TBP-TFIIA-DNA complex, TFIIA dimerization, or in vivo stability of TFIIA, suggesting this TFIIA mutant was defective in protein-protein interaction with some other component of the transcription machinery. Later work also showed the importance of other nearby residues on this TFIIA NTD surface. Protein-protein interaction studies using the two-hybrid and GST-pulldown assays suggested that the TFIIA NTD interacts with the TFIID-specific subunit Taf11 (Kraemer et al. 2001). This interaction is blocked by mutation of Toa2 residue I27, which also causes a defect in transcription from a subset of genes in vivo (Kraemer et al. 2001). In other work, it was proposed that this TFIIA NTD surface is important for transcription activation because mutation of Toa2 residue G30 suppresses the TBP mutation P109A, which is defective in activation of specific promoters (Liu et al. 1999).

To more precisely define the function of the TFIIA NTD, we used site-directed mutagenesis to define the functional surface of the TFIIA NTD. Mutations were made in plasmids containing TOA1 or TOA2 and assayed for viability and in vivo phenotype by plasmid shuffle (Table 1). Of the 14 residues mutagenized, 7 displayed in vivo phenotypes and are clustered on a conserved surface of the NTD near the tip of the four-helix bundle (Fig. 1A), with side chains conferring an in vivo phenotype colored blue and those with no phenotype colored red. Residues I27, L23, and D24, all of which show an in vivo phenotype when truncated to alanine, are conserved from yeast to human (Fig. 1B). Other nearby nonconserved residues such as V20 and E33 show an in vivo phenotype only when substituted with a large dissimilar side chain. This functional surface is bounded 
Table 1. TFIIA mutant summary

\begin{tabular}{lll}
\hline Mutant & Phenotype on plasmid & Phenotype in genome \\
\hline toa2 & & \\
N17A & wt & \\
N17L & wt & wt \\
V20A & wt & - \\
V20R & ts & ts \\
D21A & wt & dead at 36 \\
D21L & slightly ts & ts \\
L23A & ts & severe ts \\
D24A & ts & \\
D24L & severe ts & \\
T25A & wt & \\
T25L & wt & \\
I27A & slightly ts & \\
I27L & wt & \\
S28A & wt & \\
S28L & wt & \\
D29A & wt & \\
D29L & wt & \\
R31A & wt & \\
E33A & wt & \\
E33L & ts & \\
A34L & slightly ts & \\
M38A & wt & \\
M38L & wt & \\
M38R & wt & \\
toa1 & & wt \\
E235A & wt & \\
E235L & tow & \\
\hline
\end{tabular}

Phenotypes of TFIIA NTD mutants. Yeast strains containing either plasmid or integrated copies of TOA1 or TOA2 point mutants were checked for phenotypes at $18^{\circ} \mathrm{C}, 25^{\circ} \mathrm{C}, 30^{\circ} \mathrm{C}$, and $36^{\circ} \mathrm{C}$ by streaking onto glucose complete-Leu media or YPD + Ade media respectively. Two of the eight mutations were unable to integrate into the genome, indicated by -

by seven Toa1 and Toa2 residues that show no phenotype when mutated. Six of the Toa2 mutations with a defective in vivo phenotype on plasmids were integrated into the chromosomal TOA2 locus, and in all cases showed similar or, in most cases, more severe in vivo phenotypes (Table 1). An interactive 3D structure highlighting functionally important residues in the TFIIA NTD can be viewed at http://www.fhcrc.org/labs/hahn/ chime_pages/3dstruct_index.html.

To determine the biochemical defect of these mutations, we assayed the Toa2 mutations V20R and D24L for the ability to form PICs and to support transcription in vitro. TFIIA function was measured by supplementing a nuclear extract defective in TFIIA activity with recombinant TFIIA and assaying PIC formation on the yeast HIS4 promoter. The rate of PIC formation was measured by incubation of the TFIIA supplemented extract with DNA for various times, followed by nucleotide addition for a limited time to permit a single round of transcription initiation (Fig. 1C). By this assay, the absence of TFIIA resulted in about a threefold slower rate of PIC formation. Similarly, extracts containing Toa2 mutations D24L or V20R showed a defect in the rate of PIC formation with a rate twofold slower than that of wild type. Western blot analysis of PICs formed with either wild-type or mutant TFIIA showed similar relative amounts of all general transcription factors tested, suggesting that PICs formed with the TFIIA mutants were not specifically depleted in any one general factor (data not shown).

\section{Site-specific photocross-linking of PICs identifies three polypeptides near the TFIIA NTD}

Because these results, along with previous work, suggested that the TFIIA NTD mutants are defective in protein-protein interaction with some component of the transcription machinery, we set out to determine what polypeptides are located near this surface when TFIIA is assembled in PICs. In an initial approach, we isolated suppressors of Toa2 L23A and D24A by using EMS mutagenesis. Genetic analysis of these suppressors proved difficult because the phenotype of the original mutations reverted with a relatively high frequency and because the phenotype of most suppressors was weak semidominant suppression and sporulation defective. Because this genetic approach proved difficult, we directly identified polypeptides near this surface by using site-specific photocross-linking. In this strategy, TFIIA was engineered to contain unique surface accessible cysteine residues near the TFIIA functional surface, and these residues were used to attach the ${ }^{125}$ I-labeled photocross-linker PEAS, which has a probe length of $16 \AA$ (Chen et al. 1994). PICs containing this modified recombinant TFIIA were isolated and treated with UV light to activate the crosslinker. Reducing the product of this reaction with DTT transfers the labeled cross-linker from TFIIA to the cross-linked polypeptide.

Two residues near the TFIIA functional surface that showed no in vivo phenotype when mutated were individually changed to cysteine (Toal E235C and Toa2 M38C). Two surface cysteines on Toa2 were also altered (C72V and C116S) to eliminate other potential sites of cross-linker attachment. None of these changes led to an in vivo or in vitro phenotype. The photocross-linker ${ }^{125} \mathrm{I}$ PEAS was conjugated to modified Toal or Toa2 at position 235 or 38. These TFIIA derivatives were active for in vitro transcription, with activity comparable to that of wild-type TFIIA (Supplementary Fig. 1A). When PICs were formed with purified ${ }^{125}$ I-PEAS-TFIIA, activated by UV light, and reduced by DTT, the ${ }^{125}$ I-PEAS was transferred to several polypeptides (Fig. 2A, lanes 2,4). When PEAS is attached to Toa2 M38C, labeled cross-linker is transferred to a protein with apparent mobility of $65 \mathrm{kD}$ (p65) as well as to Toa1 and intramolecularly to Toa2, remaining stably bound to Toa2 after UV and DTT treatment. When cross-linker is attached to Toa1 E235C, UV and DTT treatment causes transfer to Toa2, Toa1, and proteins of $\sim 26,47$, and $65 \mathrm{kD}$. Other labeled polypeptides were not analyzed further because they were observed to be labeled in the absence of UV treatment (presumably due to the result of cys-PEAS reaction with the cysteine side chain of some other polypeptide in the ex- 
A

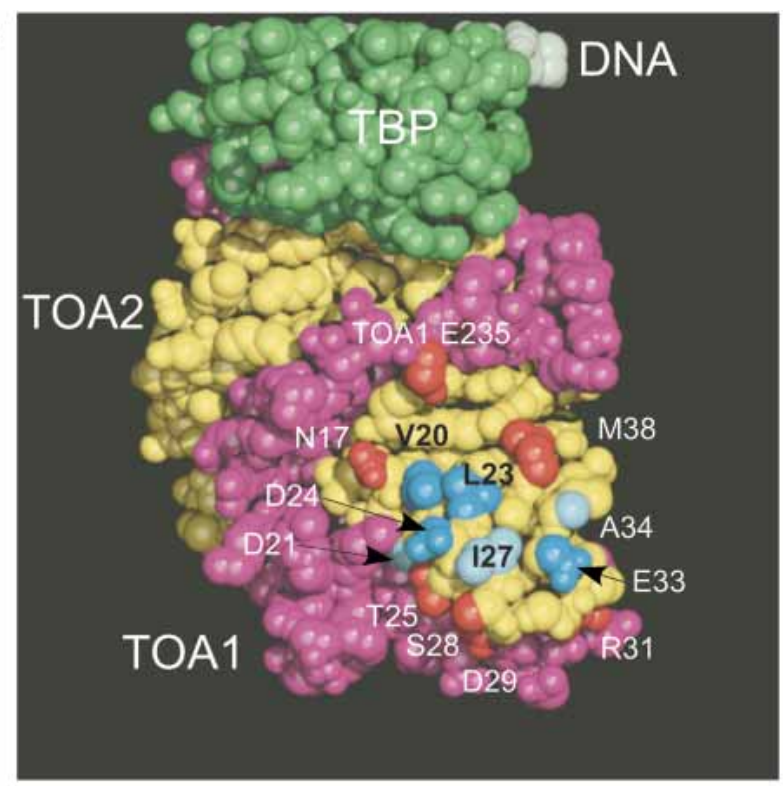

B S. cerev
S.pombe
M.griesa
N. crassa
A. thaliana
D.melano
X.laevis
M.musculus
H.sapiens 1 10 20 30 40 50 MAVPG---YYELYRRSTIGNSLVDALDTLISDGRIEASLAMRVLETFDKVVAETLKDNTQ -----MSQYYELYRRS--RISLTDALDDLISQGKISPQLAMKVLFNFDKSMTEALAEKVR MATKKEETFYELYRRTSLGICLTDALDDLITNDRINPQLAMKI LANFDRVVAETLQEKVK MAANGNTNYYDLYRHGSLGSTLTDALDDLIGAERIDPQLAMKVLMQFDRVITEALSEKVK -----MATEELYRRSTIGMCLTETLDEMVQSGTLSPELAIQVLVQFDKSMTEALESQVK -----MSYQLYRNTTLGNTLQESLDELIQYGQITPGLAFKVLLQFDKSINNALNQRVK -----MAYQLYRNTTLGNSLOESLDELIQSOQINPQLALOVLLQFDKAINSALAQRVR -- --MAYQLYRNTTLGNSLQESLDELIQSQQITPQLALQVLLQFDKAINSALAQRVR -----MAYQLYRNTTLGNSLQESLDELIQSQQITPQLALQVLLQFDKAINAALAQRVR

\section{C}

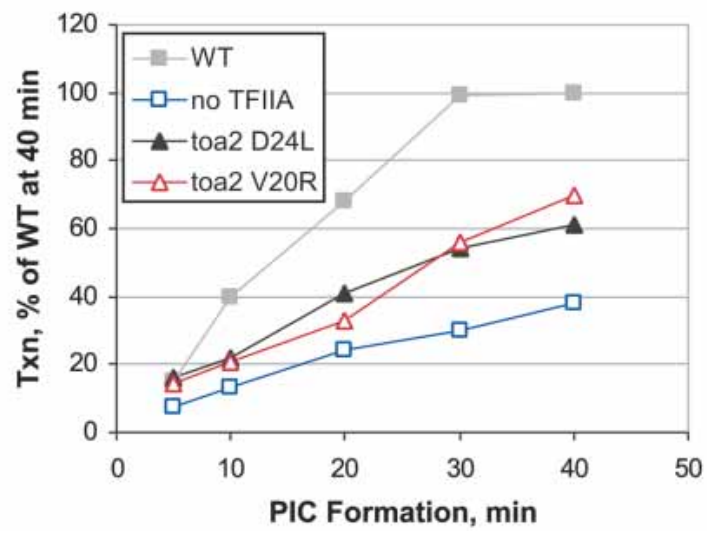

Figure 1. Functional surface of the TFIIA NTD defined by mutagenesis. (A) TBP, colored green, is bound to TFIIA where Toal is purple and Toa2 is yellow. Side chains in the $\mathrm{N}$-terminal domain of TFIIA that show no in vivo phenotype when mutated are colored red, weak phenotypes are light blue, and strong phenotypes are dark blue. All residues shown are in Toa2 except for Toal E235. Specific mutations and phenotypes are presented in Table $1 .(B)$ Conservation of residues within the TFIIA NTD. Residues 1-57 of the Saccharomyces cerevisiae Toa2 subunit are shown, as well as alignment with Toa2 from other eukaryotes. Conserved residues are highlighted. (C) TFIIA mutants have slower rates of PIC formation. PICs were formed for $5,10,20,30$, or $40 \mathrm{~min}$ in $90 \mu \mathrm{g}$ toa $1-25$ yeast nuclear extract with $40 \mathrm{ng}$ purified TFIIA-Flag ${ }_{2}$ and $30 \mathrm{ng}$ Gal4-AH on the pSH515 plasmid template, which includes $300 \mathrm{bp}$ of the HIS4 promoter and a single Gal4 site. NTPs were added and reactions stopped after 2 min to allow for a single round of transcription. Transcription activity at each time point is given as a percent of wild-type transcription with PICs formed for $40 \mathrm{~min}$. tract), or because they were not seen reproducibly (labeled with asterisks in Fig. 2A). In summary, the crosslinking assay shows that in PICs, three polypeptides with apparent mobilities of 26,47 , and $65 \mathrm{kD}$ are located within $16 \AA$ of the TFIIA NTD functional surface.

Interestingly, the cross-linking to p65 is largely activator dependent (Fig. 2B). Cross-linking to p65 is barely visible when the weaker activator Gal4-AH is used in PIC formation (Fig. 2B, lane 2) but cross-linking is strikingly stimulated when Gal4-VP16 is used to promote PIC formation (Fig. 2B, lane 4). This 18-fold stimulation is much more than can be accounted for by the 3.4-fold increase in PIC formation seen with Gal4-VP16 compared with Gal4-AH (Fig. 2B; data not shown). In contrast, cross-linking to both p26 and p47 seen with
PEAS-Toa1 E235C is not activator dependent when either Gal4-AH or Gal4-VP16 is used (data not shown).

Identification of polypeptides that cross-link to the TFIIA NTD

To identify the three cross-linked polypeptides, we first tested whether cross-linking occurs in PICs blocked at intermediate steps in assembly. Previously it was shown that PIC assembly occurs in at least three stages in this in vitro system, with TFIID and TFIIA recruited first to the promoter; TFIIB, Pol II, TFIIF, Mediator, and TFIIE recruited cooperatively in the next step; and TFIIH binding last (Ranish et al. 1999; Yudkovsky et al. 2000). PICs were assembled on immobilized templates by using ex- 
A

Figure 2. Photocross-linking of factors to the TFIIA NTD. (A) PICs were formed in a toa1-25 nuclear extract with $\sim 160 \mathrm{ng}{ }^{125}$ I-PEAS-TFIIA, where ${ }^{125} \mathrm{I}$ PEAS is conjugated to the indicated cysteine and 96 ng Gal4-VP16 on an immobilized pSH515 promoter template. Samples were treated with UV and DTT as indicated and the polypeptides were separated on SDS-PAGE and visualized by PhosphorImager. Specific cross-linked polypeptides run at 26, 47, and 65 $\mathrm{kD}$. Asterisks indicate inconsistently cross-linked polypeptides. (B) PICs were formed as in panel $A$ except in an srb2 $\Delta$ nuclear extract in the absence of recombinant Srb2 and with either 120 ng Gal4-AH or $96 \mathrm{ng}$ Gal4-VP16. $\mathrm{M}$ is the ${ }^{14} \mathrm{C}$ molecular weight marker (Sigma). (C) PICs were formed as in panel $A$ in indicated nuclear extracts with 120 ng Gal4-AH and the indicated recombinant proteins.

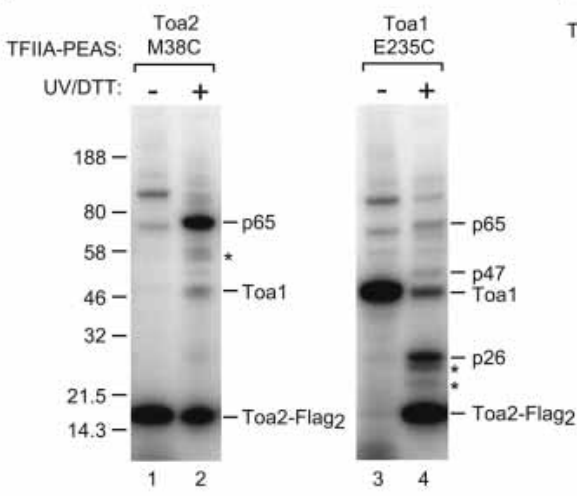

B

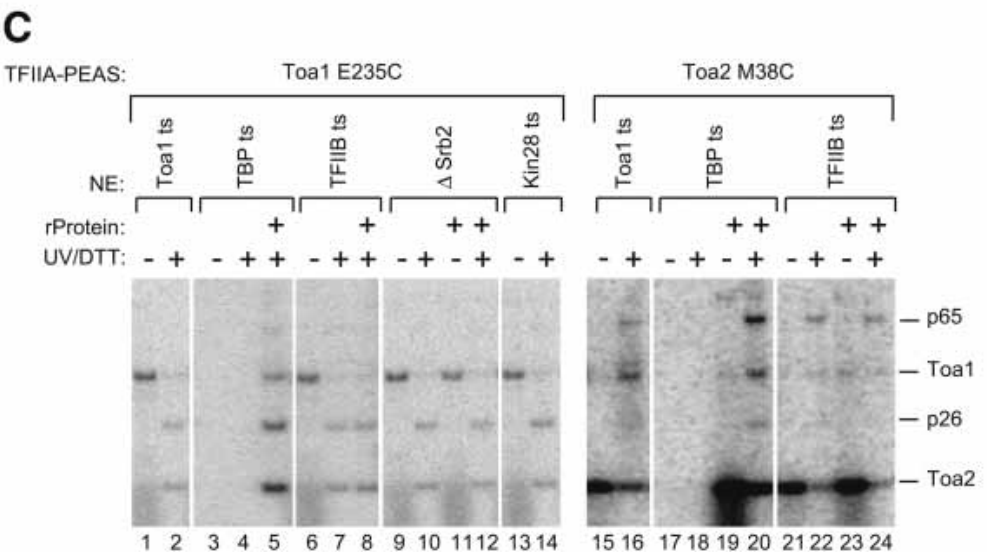

tracts containing conditional mutations in TBP, TFIIB, Mediator, or TFIIH (Fig. 2C). As expected, the observed cross-linking is TBP dependent because TFIIA is not recruited to the promoter in the absence of TBP (Fig. 2C, lanes $4,5,19,20)$. Using PEAS attached to Toal E235C, we found that cross-linking to $\mathrm{p} 26$ was not dependent on TFIIB (Fig. 2C, lane 7), Mediator (Fig. 2C, lane 10), or the CAK subcomplex of TFIIH (Fig. 2C, lane 14). Cross-linking to p47 is the weakest specific cross-linking signal observed (Fig. 2A) and was not visible in this experiment. Using Toa2 M38C-PEAS, we observed that cross-linking to p65 was also not dependent on TFIIB (Fig. 2C, lane 22). These results demonstrate that the cross-linking targets p26 and p65 are recruited during an early step in PIC formation because they are present in PICs lacking TFIIB and all other later associating factors.

With a narrowed list of possible candidates for crosslinking targets, 22 strains were constructed, each containing a triple Flag tag at the $\mathrm{C}$ terminus of a gene encoding a protein previously implicated in transcription, with a predicted size within $\sim 5 \mathrm{kD}$ of the cross-linked factors. TBP was tagged at the $\mathrm{N}$ terminus because tagging at the $\mathrm{C}$ terminus is detrimental to protein function. If PEAS is transferred to a Flag-tagged polypeptide in the PIC, then the mobility of the cross-linked polypeptide should be shifted by $5 \mathrm{kD}$ (the mobility of the $3 \times$-Flag tag in our SDS-PAGE system) compared with the untagged polypeptide (Chen and Hahn 2003; Liu et al. 2004). Using PICs made from these Flag-tagged strains, we identified p65 as Spt8 (Fig. 3A; Supplementary Fig. 2), p26 as TBP (Fig. 3B), and p47 as Taf4 (Fig. 3C). As a

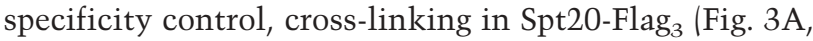
lane 3; Supplementary Fig. 2, lane 4) and Taf5-Flag (Fig. 3C, lane 3) extracts shows the same mobility of crosslinked product as compared with nontagged extracts. As expected from the structure of TBP-TFIIA-DNA complex, where the TBP conserved C-terminal domain is within $16 \AA$ of Toa1 E235 (Fig. 1A), cross-linking to TBP was not dependent on the nonconserved NTD of TBP (data not shown). Further, cross-linking to TBP was observed when purified Gal4-VP16, TBP, and PEAS-TFIIA were bound to immobilized templates in the absence of extract (data not shown). These experiments show that, in addition to TBP, a subunit of SAGA (Spt8) and TFIID (Taf4) are in close proximity to the TFIIA NTD when assembled in PICs.

These cross-linking data were derived from PEAS attached to two nearby residues on one side of the functional NTD surface. To test whether other polypeptides interacted with another surface of the NTD, we attached cross-linker to four residues on the opposite side of this cluster (D21, T25, D29, or R31; Fig. 1A). Purified TFIIA containing any of these mutations was active for transcription at near wild-type levels both before and after labeling with PEAS (Supplementary Fig. 1A). PEAS at all four positions cross-linked to Taf4 at equal or lesser intensity than PEAS-Toa1 E235C. However, none of these four new TFIIA derivatives cross-linked to either TBP or 

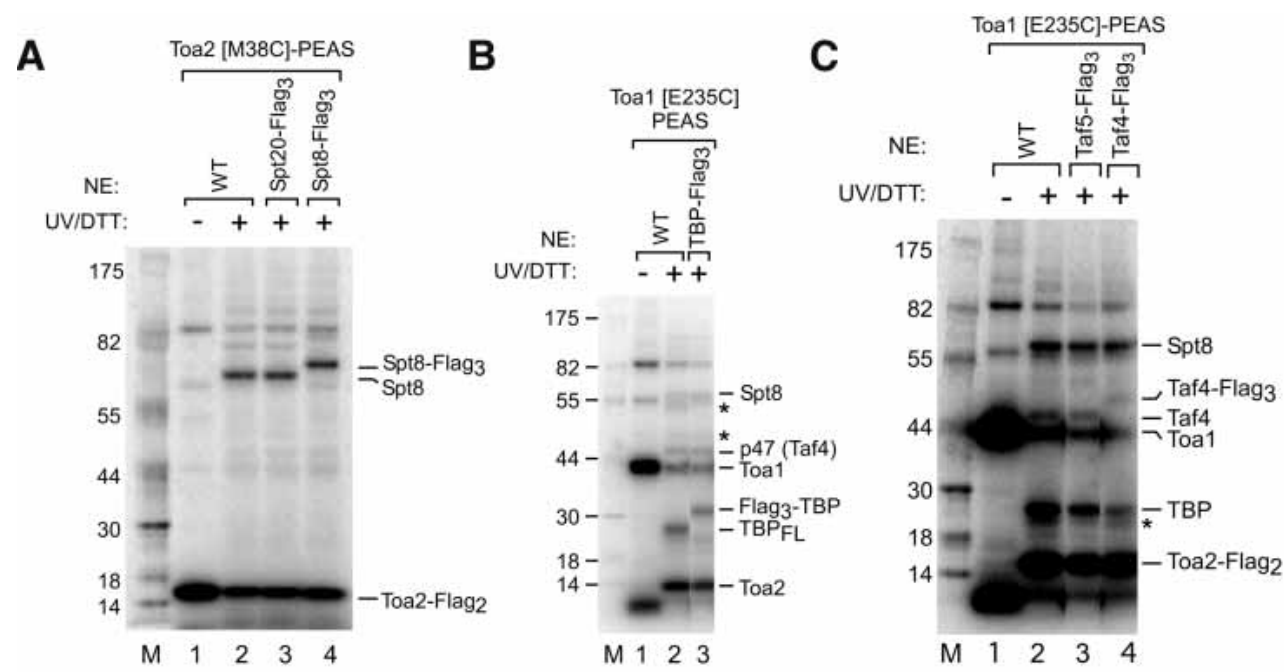

Figure 3. Spt8, Taf4, and TBP are identified as cross-linked polypeptides by using Flag-tagged nuclear extracts. $(A-C)$ PICs formed as in Figure 2A except in the indicated nuclear extracts. Asterisks indicate inconsistently cross-linked polypeptides.

Spt8, or consistently to any other polypeptide (data not shown). These data approximate the position of Spt8 and Taf4 in the PIC. Numerous other Flag-tagged nuclear extracts were also tested in the cross-linking assay, none of which displayed cross-linked polypeptides of shifted mobility. Proteins of note that did not cross-link to the TFIIA NTD include Taf11, previously shown to interact with the TFIIA NTD by two-hybrid assay (Kraemer et al. 2001), and Spt3, which genetically interacts with Spt8 and TBP in the SAGA complex (Eisenmann et al. 1994).

\section{Mapping TFIIA interaction to Spt8}

by hydroxyl radical cleavage

To further explore the interaction of TFIIA and Spt8, we first mapped the region of Spt8 located near the TFIIA NTD. To accomplish this, we used the site-specific protein cleavage reagent FeBABE (Datwyler and Meares 2000; Chen and Hahn 2003). In this strategy, the $12 \AA$ long FeBABE reagent is attached to surface-exposed cysteines, and these TFIIA derivatives are used to assemble PICs, as was described for the PEAS cross-linker. After PIC assembly, hydroxyl radicals are generated by addition of sodium ascorbate and hydrogen peroxide, leading to cleavage of the polypeptide backbone in nearby proteins. Because the hydroxyl radical can diffuse to a limited extent, this reagent probes for proteins located within $\sim 15-20 \AA$ of the Fe-EDTA center (Datwyler and Meares 2000; Chen and Hahn 2003).

FeBABE was conjugated to the same purified TFIIA cysteine mutants used in the cross-linking experiments. These TFIIA-FeBABE conjugates showed transcription activity similar to that of wild-type TFIIA (Supplementary Fig. 1B). PICs were formed by using an extract made from a strain containing $\mathrm{N}$-terminal triple Flag-tagged Spt8. Cleavage products were visualized by Western blot, probing for the Flag epitope. Both full-length Spt8 and cleavage products containing an intact $\mathrm{N}$ terminus are detected. Figure 4A shows that attempted FeBABE conjugation to TFIIA with no surface-accessible cysteines generates only full-length Flag $_{3}$-Spt 8 with no cleavage products (Fig. 4A, lanes 1,2). In contrast, FeBABE linked to six positions on either Toal or Toa2 all give unique $\mathrm{N}$-terminal cleavage products only when ascorbate and $\mathrm{H}_{2} \mathrm{O}_{2}$ are added (Fig. 4A, lanes 3-14; indicated by brackets). Although the cross-linking data showed that PEAS at Toa2 M38C and Toa1 E235C cross-links to Spt8 but PEAS-Toa2 D21C, T25C, D29C, and R31C do not, the cleavage data show that FeBABE attachment at all six TFIIA sites leads to Spt8 cleavage. This variation is likely due to the difference in the effective lengths of the two probes.

To accurately determine the location of the protein cleavage sites, we expressed a set of $\mathrm{Flag}_{3}$-Spt8 fragments by in vitro transcription and translation (Supplementary Fig. 3). The mobility of these in vitro translated products was determined by SDS-PAGE, and this was used to calculate the sizes of the $\mathrm{Flag}_{3}$-Spt8 cleavage products from Figure 4A. From multiple mapping experiments and from mapping some cleavage sites from both ends of Spt8, we estimate that these cleavage sites are accurate to within \pm 5 amino acids. The Spt 8 cleavage sites determined by this method are shown in Figure 4B, with the length of the line representing relative cleavage efficiency. The cleavage products from Figure 4A map to the C-terminal half of Spt8, and the more intense cleavage products are clustered near the $\mathrm{C}$ terminus. Supplementary Table 1 summarizes the Toa1 and Toa2 cysteine mutations used in the FeBABE mapping, the residues on Spt8 cleaved by these mutants, and the intensity of the Flag $_{3}$-Spt8 cleavage products produced. Although these cleavage sites are spread over almost 300 residues, their positions are likely in close proximity in the $3 \mathrm{D}$ structure of Spt8 (Chen and Hahn 2003).

We also attempted to map Taf4 interaction with TFIIA by the FeBABE cleavage assay; however, no cleavage 
Warfield et al.

A

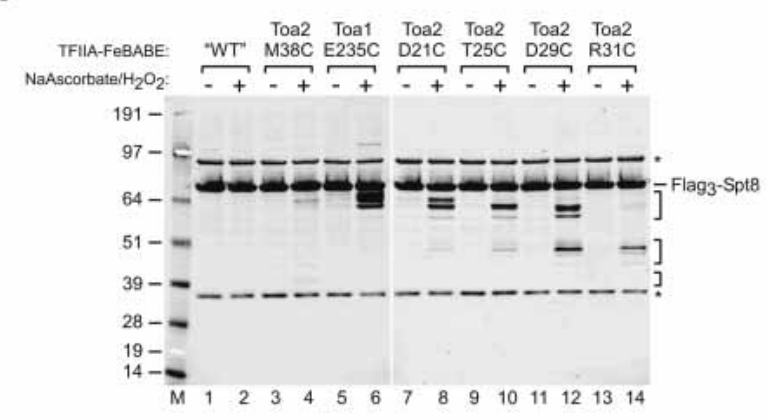

B

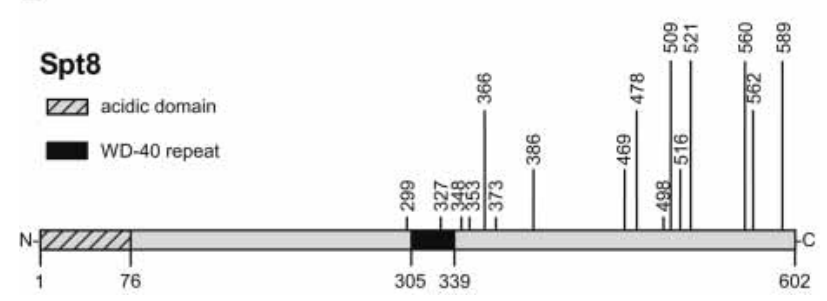

Figure 4. Determination of TFIIA-FeBABE cleavage sites in Spt8. (A) Western blot analysis of TFIIA-FeBABE cleavage of Flag $_{3}$-Spt8 probed with $\alpha$-Flag. PICs were formed as in Figure 2A except in a Flag ${ }_{3}$-Spt8 nuclear extract with $\sim 200$ ng TFIIA conjugated with FeBABE on the indicated cysteines. TFIIA with no surface cysteines after attempted FeBABE conjugation is indicated by "WT". Hydroxyl radical cleavage was initiated with the addition of $\mathrm{Na}$ Ascorbate and $\mathrm{H}_{2} \mathrm{O}_{2}$ where indicated. Brackets indicate cleavage products and asterisks indicate nonspecific signals. (B) Map of Spt8 with the TFIIA-FeBABE cleavage sites listed in Supplementary Table 1. The length of the line indicating each cleavage site corresponds to the intensity of the band from panel $A$. Positions of the acidic domain and the WD-40 repeat are indicated.

products were observed (data not shown). One possible explanation for this result is that FeBABE cleavage of $\mathrm{Flag}_{3}$-Taf4 occurs very near the $\mathrm{N}$ or $\mathrm{C}$ terminus of Taf4. Cleavage very near the $\mathrm{N}$ terminus would generate fragments too small to observe by SDS-PAGE, and cleavage very near the $\mathrm{C}$ terminus would generate fragments too large to separate from full-length $\mathrm{Flag}_{3}$-Taf4. Additionally, because cross-linking of TFIIA to Spt8 is much stronger than to Taf4, the NTD of TFIIA is almost certainly closer to Spt8 in the PIC compared with Taf4.

\section{Genetic interaction between TFIIA, Spt8, and Spt3}

To independently confirm the interaction of TFIIA and Spt8, we tested whether these two factors interact genetically. In this strategy, a strain containing a double mutation in both the TFIIA NTD and Spt 8 was tested for an in vivo phenotype that was stronger than the phenotype of either single mutation. We used a similar assay to test for genetic interaction between Spt3 (genetically related to Spt8) and the TFIIA NTD. Yeast strains containing an spt8s and either wild-type TOA2, toa2 L23A, or toa2 D24L were constructed and tested for growth on rich media at $25^{\circ} \mathrm{C}$ and $37^{\circ} \mathrm{C}$. Figure 5 shows that neither SPT3 nor SPT8 deletions display a growth phenotype under these conditions. In contrast, both TFIIA NTD mutants show temperature-sensitive growth at $37^{\circ} \mathrm{C}$. When these TFIIA NTD mutations are combined with either $s p t 3 \Delta$ or spt $8 \Delta$ deletions, the strains display a mild slow growth phenotype at $25^{\circ} \mathrm{C}$ and a stronger temperaturesensitive phenotype at $37^{\circ} \mathrm{C}$ compared with the NTD mutations alone. These results demonstrate a genetic interaction between the NTD of TFIIA and the two SAGA subunits Spt 3 and Spt8 and are consistent with previous findings of a genetic interaction between TFIIA and Spt3 (Madison and Winston 1997).

\section{Spt8 and TFIIA compete for binding to TBP}

One model to explain these data is that the $\mathrm{C}$ terminus of Spt8 binds to the TFIIA NTD, providing a direct connection between SAGA and the general transcription factors. This model was tested directly by using recombinant TFIIA, TBP, and Spt8 (Fig. 6A). Purified GST or GST-Spt 8 was bound to glutathione Sepharose beads and incubated with TBP, TFIIA, or both TBP and TFIIA. No significant amount of TBP or TFIIA bound to GST alone (Fig. 6A, lanes 5,7,9). TBP bound strongly to GST-Spt8, consistent with previous findings that SAGA binding to TBP was dependent on Spt8 (Fig. 6A, lanes 6,8; Sterner et al. 1999). TBP also bound a C-terminal construct of Spt8 (residues 265-602) to the same extent as full-length Spt8 (data not shown). Surprisingly, TFIIA did not bind to either full-length GST-Spt8 or GST-Spt8 (265-602) (Fig. 6A, lanes 8,10; data not shown). Strikingly, when both TBP
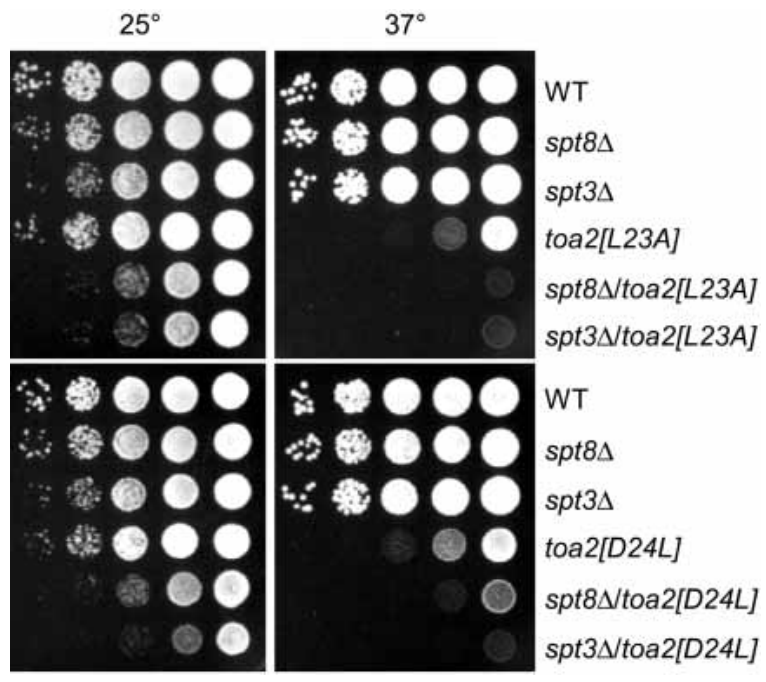

Figure 5. Genetic interaction between Spt8, Spt3, and TFIIA Strains were grown in YPD + adenine overnight at $30^{\circ} \mathrm{C}$ and diluted to $5 \times 10^{7}$ cells $/ \mathrm{mL}$, and $3.5 \mu \mathrm{L}$ of 10 -fold dilutions were spotted to YPD + adenine plates and grown for $2 \mathrm{~d}$ at $25^{\circ} \mathrm{C}$ or $37^{\circ} \mathrm{C}$. 

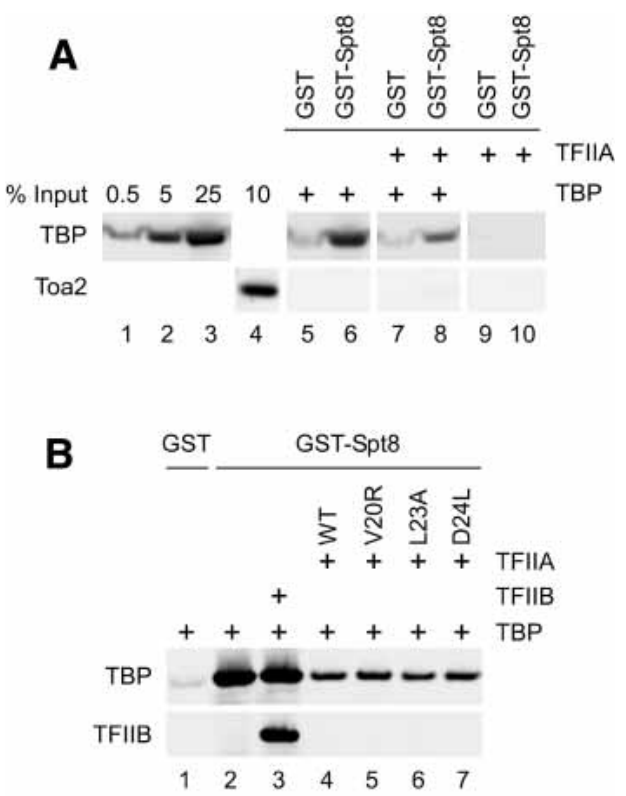

Figure 6. Spt8 competes with TFIIA for binding to TBP. (A) Purified GST or GST-Spt 8 bound to glutathione sepharose beads was incubated with recombinant full-length TBP and/or a sevenfold excess of TFIIA. After separation by SDS-PAGE, the Western blot was probed with $\alpha$-TBP and $\alpha$-Toa2. Known amounts of purified TBP and TFIIA were run in lanes 1-4. GST and GST-Spt8 were eluted, separated by SDS-PAGE, and Coomassie stained to confirm that similar amounts of GST and GST-Spt 8 were bound to the beads (data not shown). (B) Purified full-length TBP and a sevenfold excess of either TFIIB, wild-type TFIIA, or TFIIA NTD mutants were incubated with GST or GST-Spt 8 as indicated earlier, then bound protein was analyzed by Western blot with $\alpha$-TBP and $\alpha$-TFIIB as described in panel $A$. The TFIIA point mutations indicated are each in the Toa2 subunit. TFIIB does not significantly bind to GST beads (not shown).

and either a twofold or sevenfold excess of TFIIA were present in the reaction, TFIIA was found to inhibit TBP binding to GST-Spt8 (Fig. 6A, lanes 6,8; data not shown). Using known amounts of TBP to quantitate TBP binding (Fig. 6A, lanes 1-3), we found that TFIIA inhibited TBP binding to Spt8 13-fold under these in vitro conditions. In similar assays, no binding was detected when Spt3$\mathrm{His}_{6}$ was tested for binding to GST-Spt8, suggesting Spt3 and Spt8 do not interact directly under these conditions (data not shown).

To test whether the competition between Spt8 and TFIIA was specific for TFIIA, we tested whether TFIIB, another TBP-interacting factor, would compete with Spt8 for TBP binding (Fig. 6B). TBP with or without a sevenfold molar excess of yeast TFIIB was incubated with GST-Spt8 beads as described earlier, washed, and analyzed for factors binding to the immobilized Spt8 beads. TBP interacting with Spt8 clearly bound TFIIB, and this Spt8 binding was not inhibited by TFIIB (Fig. 6B, lanes 2,3). In contrast, a sevenfold excess of TFIIA strongly inhibited TBP binding to Spt8 and this inhibi- tion was not affected by mutations in the TFIIA NTD that inhibit the positive function of the NTD. From these experiments, we conclude that inhibition of Spt8 binding is specific to TFIIA and that the inhibitory surface of TFIIA is not limited to one face of the TFIIA NTD.

Although these results show that purified TFIIA and Spt8 compete for binding to TBP, cross-linking and other biochemical results shown earlier demonstrate that TBP, Spt8, and TFIIA are simultaneously present and are in close proximity within the PIC. Together, these results suggest that the presence of Spt8 in the SAGA complex may reduce the affinity of SAGA binding to the general transcription factors because of competition with TFIIA. To test this model, we assayed whether deletion of Spt8 results in more efficient binding of SAGA to the PIC. One complication in testing this model is that in vivo studies have shown that SAGA can be recruited to promoters via interaction with the activators GAL4 and VP16 independently of the transcription machinery (Bhaumik and Green 2001). Binding to VP16 is also independent of the general factors in the in vitro transcription system (Fig. 7A). A low amount of SAGA is found in PICs formed in the absence of any activator (Fig. 7A, lane $1)$, and recruitment of SAGA is greatly stimulated by Gal4-VP16 binding to a single site upstream of the core

A

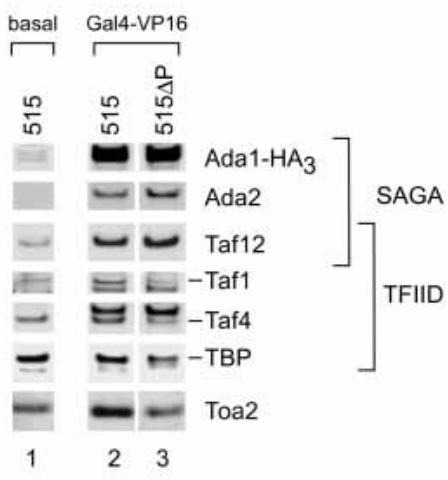

B

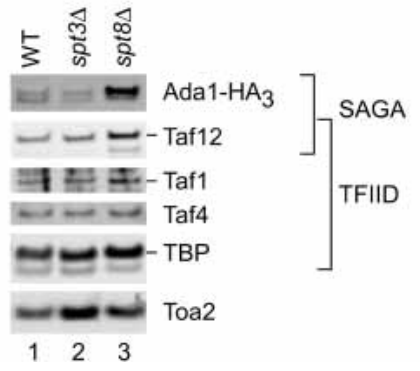

Figure 7. Interaction of SAGA with activator and the basal transcription machinery. (A) Western blot of PICs formed in WT nuclear extracts on either immobilized pSH515 template or pSH515 $\mathrm{P}$ template, which contains $150 \mathrm{bp}$ of nonpromoter sequence and a single Gal4 binding site. Either no activator or Gal4-VP16 was added as indicated. (B) Western blot of PICs formed in the indicated nuclear extracts with no activator. 
promoter (Fig. 7A, lane 2). Consistent with in vivo results, the stimulation of recruitment by VP16 does not require an intact core promoter. When an immobilized template containing the activator binding site but lacking the core promoter is used $(515 \Delta \mathrm{P})$, the TFIID-specific subunits Taf1 and Taf4, as well as TBP and TFIIA, are found at lower levels compared with the intact promoter (Fig. 7A, lanes 2,3). In contrast, SAGA-specific subunits Ada1, Ada2, or Taf12 (shared between TFIID and SAGA) are insensitive to promoter deletion, suggesting that SAGA binds to the activator independently of both the general factors and promoter DNA. Consistent with this conclusion, SAGA is recruited to the immobilized template in an activator-dependent fashion in extracts lacking functional TBP that are blocked in the first step in PIC assembly (Yudkovsky 2001).

To assay the direct interaction of SAGA with the general factors, we monitored SAGA binding to PICs in the absence of any added activator. Levels of the SAGA-specific subunit Adal and the TFIID/SAGA subunit Taf12 present in the PIC were compared in wild-type, spt3s, and spt $8 \Delta$ extracts by quantitation of Western blot signals (Fig. 7B). As predicted earlier, SAGA lacking the Spt8 subunit was recruited to the PIC an average of 3.3fold better compared with wild-type extracts in three separate experiments. The level of Taf12, a shared subunit between SAGA and TFIID, was also increased in the spt $8 \Delta$ extract. In contrast, all other non-SAGA subunits tested showed no reproducible change when all three extracts were compared. These results demonstrate that within the SAGA complex, Spt8 has a negative effect on SAGA interaction with the general factors under basal transcription conditions. This is consistent with the previous finding that $\mathrm{Spt} 8$ inhibits basal expression from the HIS3 and TRP3 promoters in vivo (Belotserkovskaya et al. 2000). In contrast, SAGA lacking Spt3 was recruited slightly less well, with an average twofold lower Adal level in PICs compared with wild type (Fig. 7B, lanes 1,2 ).

Figure 8. The function of the TFIIA NTD in enhancing the rate of PIC formation is mediated largely through Spt8. (A, left panel) Single-round transcription assayed by primer extension. PICs were formed for the indicated times by using $90 \mu \mathrm{g}$ of spt8s, Toa2-Flag nuclear extract, which was depleted of TFIIA by using $\alpha$-Flag M2 agarose (Sigma) and supplemented with a saturating amount $(20 \mathrm{ng})$ of recombinant TFIIA. NTPs were added and reactions stopped after 2 min to allow for a single round of transcription. (Right panel) Multiround transcription assayed by primer extension by using $90 \mu \mathrm{g}$ of either wild-type extract or the spt8s extract depleted for TFIIA as described earlier and supplemented with 20 ng purified "wild-type" TFIIA. All purified TFIIA proteins used in this experiment have the surface cysteines eliminated (Toa2 C72V and C116S), and display wild-type in vitro transcription activity (Supplementary Fig. 1A). (B) Quantitation of data presented in the left side of panel $A$. Transcription activity at each time point is given as a percent of wild-type transcription with PICs formed for $40 \mathrm{~min}$.
The positive function of the TFIIA NTD is mediated through Spt8

In addition to repression of basal expression, Spt8 also positively regulates transcription at some SAGA-dependent promoters (Bhaumik and Green 2001; Larschan and Winston 2001). To test whether the positive function of the TFIIA NTD is mediated through Spt8, we determined the effect of the TFIIA NTD mutations in a strain lacking Spt8. Extracts from a $s p t 8 \Delta$ strain were found to be about threefold deficient for multiround transcription (Fig. 8A, lanes 21,22), confirming the positive role of Spt8 in the in vitro system. If the positive function of the TFIIA NTD is mediated through Spt8, then the activity of the NTD mutants should be comparable to wild-type TFIIA in a spt $8 \Delta$ extract. TFIIA was depleted from the spt8s extract and this depleted extract was supplemented by adding recombinant wild-type TFIIA or the TFIIA mutants Toa2 D24L or V20R. As shown in Figure $1 \mathrm{C}$, these TFIIA mutations result in a twofold decrease in the rate of PIC formation in an otherwise wild-type extract. In contrast, both TFIIA mutants have activity at or near the level of wild-type TFIIA in the spt8s extract (Fig. 8). Comparing the initial rate of PIC formation during the first $20 \mathrm{~min}$, we found that the TFIIA mutants Toa2 D24L and V20R support a rate of PIC formation equal to or within $80 \%$ of wild-type, respectively. This demonstrates that the positive function of the TFIIA NTD is largely mediated through Spt8 in the in vitro system. In contrast, complete lack of TFIIA still resulted in a low rate of PIC formation in the spt8s strain. This result is consistent with the importance of the TFIIA C-terminal domain in other functions such as stabilization of TBP-DNA binding and competition with inhibitors of TBP function.

\section{Discussion}

SAGA is an important cofactor for activation of a subset of genes in yeast. At SAGA-dependent promoters, SAGA

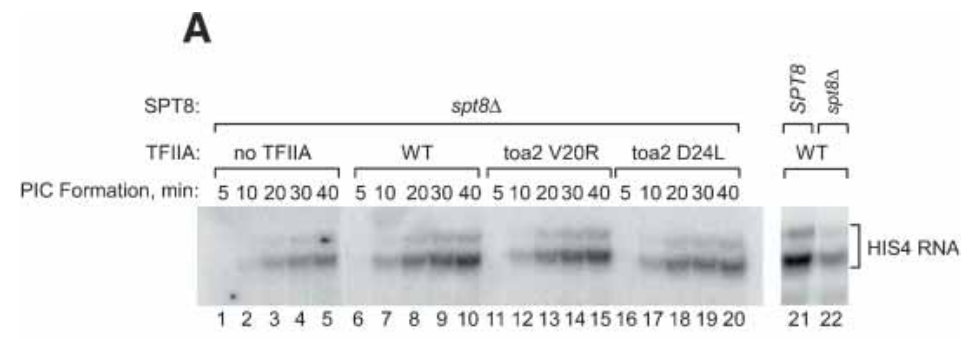

B

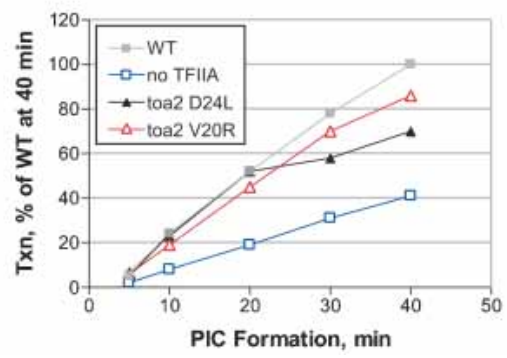


appears to be recruited directly to the activator, independently of the transcription machinery. Mutations in SAGA subunits such as Spt3 or Spt8 do not affect the recruitment of SAGA to the activator, but can block subsequent recruitment of the general transcription factors (Bhaumik and Green 2001; Larschan and Winston 2001). SAGA was also shown to repress basal expression from specific promoters in yeast, mediated by the Spt3 and Spt8 subunits. The mechanism by which SAGA interacts with the general factors has been unclear because of conflicting genetic and biochemical data. Here, we have demonstrated an unexpected connection between Spt8, TBP, and the NTD of TFIIA. When assembled into PICs, site-specific photocross-linking shows that Spt8 is closest to the functional surface of the TFIIA NTD. This NTD surface is also near the TFIID-specific subunit Taf4, but the observed weak cross-linking signal suggests that Taf4 is located near the limit of the $16 \AA$-probe distance and therefore may not directly interact with the TFIIA NTD. The dependence of Spt8-NTD cross-linking on TBP demonstrates that in PICs, TBP, TFIIA, and Spt8 are all in close proximity. No cross-linking was observed between the TFIIA NTD and Taf11, which were previously proposed to interact on the basis of the two-hybrid assay and two mutations in the TFIIA NTD that blocked this two-hybrid interaction (Kraemer et al. 2001). In contrast, our results suggest that the TFIIA NTD and Taf11 do not normally interact when assembled into PICs.

FeBABE mapping showed that at least 16 residues spread throughout the C-terminal half of Spt8 are near the TFIIA NTD when assembled in PICs. Although the structure of Spt8 is not yet known, it is likely that these FeBABE reactive residues are located in close proximity in the native Spt8 structure. The C-terminal half of Spt8 also bound TBP with the same affinity as full-length Spt8. Together, these findings suggest that the C-terminal half of Spt8 forms a domain that interacts with both TBP and the TFIIA NTD.

Given the finding that TFIIA, TBP, and Spt8 are all in close proximity in PICs, it was surprising that experiments that used purified proteins showed that Spt8 and TFIIA do not stably bind. In contrast, it was observed that both Spt8 and TFIIA compete for binding to TBP. On the basis of the cross-linking results showing that TBP, Spt8, and TFIIA are simultaneously present in PICs, we propose that $\operatorname{Spt} 8$ and TFIIA do not bind to the same location on the surface of TBP, but rather sterically interfere with each other when each interacts with its binding site on TBP. This work using purified proteins is supported by studies using the intact SAGA complex in which SAGA lacking Spt8 bound more efficiently than wild-type SAGA to a PIC. Also consistent with our results is the finding that deletion of SPT8 (or SPT3) can cause derepression of basal expression from a subset of genes in vivo such as HIS3 and TRP3 (Belotserkovskaya et al. 2000).

We have also shown that the positive function of the TFIIA NTD surface is largely mediated through Spt8 in vitro. The TFIIA NTD was demonstrated to play a role in the rate of PIC formation, because extracts with TFIIA
NTD mutations were found to form PICs with a twofold lower rate compared with wild-type extracts. Extracts lacking Spt8 were found to have about a threefold defect for transcription in vitro, and in these extracts TFIIA NTD mutants and wild-type TFIIA gave nearly equivalent rates of PIC formation. This result is consistent with a mechanism where the function of the TFIIA NTD is dependent on Spt8 activity. Together, our findings show that Spt8 can have both a positive and a negative function on transcription, mediated through TFIIA.

How can the competitive interaction between TFIIA and Spt8 lead to positive effects on gene expression? One model to explain these results is that during activated transcription, the interaction between the TFIIA NTD and $\mathrm{Spt} 8$ causes a conformational change leading to a more active state (Fig. 9). This conformational change would be driven by the binding energy derived from interaction of TFIIA and Spt8 with TBP as well as by other presumed protein-protein interactions between SAGA, activator, and the transcription machinery. It is proposed that Spt8 and/or TFIIA adopts a structure that is more efficient at promoting transcription in this altered conformation. Under basal or unactivated conditions at Spt8-dependent promoters, this proposed conformational change would not occur efficiently, leading to the repression of basal expression observed in vivo. Such a model would explain the observed genetic interaction between TFIIA and Spt8 where mutation in the TFIIA NTD combined with an $s p t 8 \Delta$ has a more severe phenotype than either single mutation. We postulate that each factor alone has an independent positive function such as promotion of TBP/TFIID binding or coactivator function. Together, both factors cooperate to generate a more active state of the transcription machinery, explaining why the phenotype of the double mutation is the most severe. Although this model is unusual, the direct photocross-linking assay can rule out more conventional com-

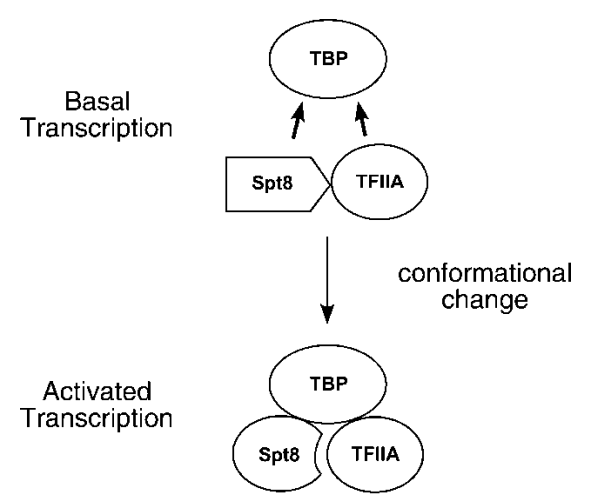

Figure 9. Proposed model of interactions among TFIIA, Spt8, and TBP. TFIIA and Spt8 each bind individually to TBP; however, they compete for TBP binding under basal transcription conditions. Because the photocross-linking results indicate that all three proteins are simultaneously present in the PIC under activated transcription conditions, a conformational change may occur in Spt8 and/or TFIIA, allowing both Spt8 and TFIIA to stably bind TBP and leading to transcriptional stimulation. 
petition mechanisms whereby PICs are assembled in vitro with either TFIIA or Spt8.

The regulation of transcription by Spt8 almost certainly does not happen at all promoters, because only a subset of promoters is SAGA dependent (Lee et al. 2000). In addition, SLIK/SALSA, a SAGA-related complex, lacks the Spt8 subunit altogether and contains at least one unique subunit, Rtg2 (Pray-Grant et al. 2002; Sterner et al. 2002; Wu and Winston 2002). Under our in vitro conditions, we do not detect significant levels of Rtg2 in PICs formed at the HIS4 promoter (G. Rani and S. Hahn, unpubl.). At some promoters in vivo, it is also possible that Spt8 plays a strictly negative role (Belotserkovskaya et al. 2000), an issue that could be addressed by genomewide expression assays. Finally, similar mechanisms involving both positive and negative protein-protein interactions may partly explain why other TBP interacting factors, such as NC2 and Mot1, inhibit assembly of the PIC with purified factors but have been shown to act positively on a subset of genes in vivo (Pereira et al. 2003).

\section{Materials and methods}

Yeast strains and nuclear extracts

Strains used in this study were as follows: BY4705 (wild type; Brachmann et al. 1998); SHY193 Mata ura3 lys2 ade2 trp1 his3 leu2 toa2A::LYS2/pSH342 (ARS CEN URA3 TOA2); SHY194, same as SHY193 but Mata; SHY195 Mata ura3 lys2 ade2 trp1 his3 leu2 toa1A::LYS2/pSH325 (ARS CEN URA3 TOA1); SHY196, same as SHY195 but Mata; SHY109 (toa1-25ts; Kang et al. 1995); SHY70 (TBP I143N ts; Reddy and Hahn 1991); SHY245 (sua7 G41E cs; Ranish et al. 1999); SHY202 (Z525) srb2d (Ranish et al. 1999); kin28-ts16 (Cismowski et al. 1995). LHY78, LHY79, SHY519, and SHY512 contain triple Flag tags at the C terminus of Spt20, Spt8, Taf5, and Taf4, respectively. LHY99 contains a triple Flag tag at the $\mathrm{N}$ terminus of TBP.

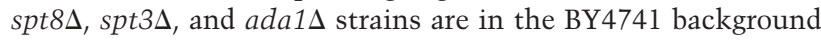
from Research Genetics; LHY4 and LHY10, respectively, contain toa2 D24L and toa2 L23A integrated at the TOA2 locus in BY4705. LHY117 (toa2 D24L, spt8s), LHY118 (toa2 D24L, spt3s), LHY119 (toa2 L23A, spt8A), and LHY120 (toa2 L23A, spt3 $\Delta$ ) were constructed by mating and sporulation of LHY4 or LHY10 with $s p t 8 \Delta$ or $s p t 3 \Delta$ strains. Yeast nuclear extracts were prepared according to the method described at http://www. fhcrc.org/labs/hahn.

\section{TFIIA mutagenesis and purification}

TFIIA mutations for the phenotype screen were generated by site-directed mutagenesis of pSH343 (2 kb PstI TOA2 fragment in pRS315) or pSH363 ( 2.2 kb BamHI/XhoI TOA1 fragment in pRS315). TOA1 and TOA2 mutants were checked for phenotype on minimal media by plasmid shuffle assay. Cysteine substitutions in Toa2 and Toa1 for PEAS and FeBABE attachment were made in pSH343 or pSH363. All TFIIA cysteine mutants also had two naturally occurring surface cysteines on Toa2 eliminated (C72V and C116S). Phenotypes of all mutations were checked by plasmid shuffle assay. TFIIA expression and purification are described at http://www.fhcrc.org/labs/hahn.

\section{PEAS and FeBABE attachment to TFIIA}

One hundred fifty micrograms $(200-400 \mu \mathrm{L})$ of purified TFIIA was exchanged into labeling buffer (30 mM Tris, 10\% glycerol,
$200 \mathrm{mM} \mathrm{KCl}$ at $\mathrm{pH} 7.4$ ) by using NAP-5 columns (Amersham) and concentrated to $\sim 30 \mu \mathrm{L}$ by using Ultrafree Biomax4-10K concentrators (Millipore). The photocross-linker PEAS \{N-[(2pyridyldithio)ethyl]-4-azidosalicylamide; Molecular Probes\} was labeled with ${ }^{125} \mathrm{I}$ (350-600 $\mathrm{mCi} / \mathrm{mL}$; Amersham) in Iodogen tubes (Pierce) containing $110 \mu \mathrm{L} 0.1 \mathrm{M} \mathrm{Na}$ Phosphate (pH 7.4), 11 nmole PEAS, and $\sim 1.5 \mathrm{mCi}{ }^{125} \mathrm{I}$ per $150 \mu \mathrm{g}$ TFIIA. After 5 min, the reaction was transferred to a tube containing 100 nmole tyrosine per TFIIA labeling to scavenge unreacted ${ }^{125} \mathrm{I}$. This mixture was supplemented with $150 \mu \mathrm{g}$ TFIIA and allowed to react for $30-45 \mathrm{~min}$ at $23^{\circ} \mathrm{C}$. ${ }^{125}$ I-PEAS-TFIIA was purified from unreacted ${ }^{125}$ I-PEAS by buffer exchange on a NAP-5 column into $1 \mathrm{~mL}$ fresh labeling buffer. Recovery was $\sim 100 \mu \mathrm{g}$ with an activity of $6-11 \mu \mathrm{Ci} / \mu \mathrm{g}$ TFIIA, whereas control TFIIA with no surface cysteines had an activity of $\sim 0.5 \mu \mathrm{Ci} / \mu \mathrm{g}$ TFIIA after attempted labeling. Labeled TFIIA was immediately aliquoted and stored at $-70^{\circ} \mathrm{C}$. TFIIA was shown to be labeled on the correct subunit by SDS-PAGE followed by autoradiography. As expected, PEAS was cleaved from TFIIA by addition of $50 \mathrm{mM}$ DTT.

FeBABE [iron(S)-1-(p-bromoacetamido-benzyl) EDTA; Do-

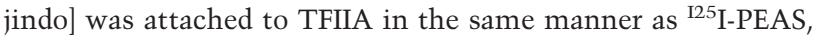
except $135 \mu$ g TFIIA was exchanged into conjugation buffer (10 $\mathrm{mM}$ MOPS, $200 \mathrm{mM} \mathrm{NaCl}, 2 \mathrm{mM}$ EDTA, $5 \%$ glycerol at $\mathrm{pH}$ 8.0 ), concentrated to $\sim 100 \mu \mathrm{L}$, and reacted with a 20 -fold molar excess of FeBABE for $4 \mathrm{~h}$ at $23^{\circ} \mathrm{C}$. The TFIIA-FeBABE was purified from unreacted FeBABE by exchange into TFIIA storage buffer $(30 \mathrm{mM}$ Tris, $200 \mathrm{mM} \mathrm{KCl}, 2 \mathrm{mM}$ EDTA, 10\% glycerol at $\mathrm{pH} 7.5)$ and immediately aliquoted and stored at $-70^{\circ} \mathrm{C}$.

Pol II immobilized template, photocross-linking, and FeBABE cleavage assays

Immobilized template assays for PIC formation, photocrosslinking, and FeBABE cleavage reactions were performed as previously described (Chen and Hahn 2003) except that 240-480 $\mu \mathrm{g}$ yeast nuclear extract was used depending on the extract (extracts were dialyzed to remove DTT for photocross-linking) and $\sim 160 \mathrm{ng}$ of either ${ }^{125}$ I-PEAS-TFIIA or FeBABE-TFIIA was added. The molecular sizes of $\mathrm{Flag}_{3}$-Spt 8 fragments generated by FeBABE cleavage were determined as previously described (Chen and Hahn 2003), using a set of in vitro translated Flag $_{3}$ Spt8 constructs. Proteins were visualized and quantitated by Western blot by using the LI-COR Bioscience Odyssey Infrared imaging system.

\section{GST pull-down assay}

One microgram GST or GST-Spt8 protein was bound per $5 \mu \mathrm{L}$ glutathione Sepharose beads in acetate transcription buffer (100 mM KOAc, $100 \mathrm{mM}$ HEPES, $1 \mathrm{mM}$ EDTA, $5 \mathrm{mM} \mathrm{MgOAc}$ at $\mathrm{pH}$ 7.6), $160 \mu \mathrm{g} / \mathrm{ml} \mathrm{PMSF}, 50 \mu \mathrm{g} / \mathrm{ml}$ BSA, $0.05 \%$ NP-40 for $1 \mathrm{~h}$ at $4^{\circ} \mathrm{C}$, then washed three times. Each pull-down reaction contained $25 \mu \mathrm{L}$ GST or GST-Spt8 beads, $1 \mu \mathrm{g}$ full-length TBP, and/or $25 \mu \mathrm{g}$ TFIIA in a total volume of $400 \mu \mathrm{L}$ of the buffer. Reactions were rotated for $1.5 \mathrm{~h}$ at $4^{\circ} \mathrm{C}$, washed three times, and then resuspended in $20 \mu \mathrm{L} 1 \times$ NuPAGE LDS sample buffer (Invitrogen) and proteins were eluted at $95^{\circ} \mathrm{C}$ for $5 \mathrm{~min}$. Proteins were visualized by Western blot.

\section{Acknowledgments}

We thank S. Hurst for initial work on mutagenesis of TFIIA, A. Movius and H-T. Chen for help with development of photocross-linking and FeBABE assays, and B. Moorefield for help 
with in vitro transcription/translation. We thank H-T. Chen, N. Mohibullah, and G. Rani for nuclear extracts; F. Winston, T. Weil, M. Green, and J. Reese for plasmids and antibodies; D. Gottschling for discussion and advice on genetic analysis of TFIIA; and B. Moorefield and W. Reeves for comments on the manuscript. This work was supported by a grant from the NIH. S.H. is an associate investigator of the Howard Hughes Medical Institute.

The publication costs of this article were defrayed in part by payment of page charges. This article must therefore be hereby marked "advertisement" in accordance with 18 USC section 1734 solely to indicate this fact.

\section{References}

Agalioti, T., Lomvardas, S., Parekh, B., Yie, J., Maniatis, T., and Thanos, D. 2000. Ordered recruitment of chromatin modifying and general transcription factors to the IFN-beta promoter. Cell 103: 667-678.

Auble, D.T., Hansen, K.E., Mueller, C.G.F., Lane, W.S., Thorner, J., and Hahn, S. 1994. Mot1, a global repressor of RNA polymerase II transcription, inhibits TBP binding to DNA by an ATP-dependent mechanism. Genes \& Dev. 8: 1920-1934.

Belotserkovskaya, R., Sterner, D.E., Deng, M., Sayre, M.H., Lieberman, P.M., and Berger, S.L. 2000. Inhibition of TATAbinding protein function by SAGA subunits Spt3 and Spt8 at Gcn4-activated promoters. Mol. Cell. Biol. 20: 634-647.

Bhaumik, S.R. and Green, M.R. 2001. SAGA is an essential in vivo target of the yeast acidic activator Gal4p. Genes \& Dev. 15: 1935-1945.

- 2002. Differential requirement of SAGA components for recruitment of TATA-box-binding protein to promoters in vivo. Mol. Cell. Biol. 22: 7365-7371.

Brachmann, C.B., Davies, A., Cost, G.J., Caputo, E., Li, J., Hieter, P., and Boeke, J.D. 1998. Designer deletion strains derived from Saccharomyces cerevisiae S288C: A useful set of strains and plasmids for PCR-mediated gene disruption and other applications. Yeast 14: 115-132.

Cang, Y., Auble, D.T., and Prelich, G. 1999. A new regulatory domain on the TATA-binding protein. EMBO J. 18: 66626671.

Chen, H.T. and Hahn, S. 2003. Binding of TFIIB to RNA polymerase II: Mapping the binding site for the TFIIB zinc ribbon domain within the preinitiation complex. Mol. Cell 12: $437-$ 447.

Chen, Y., Ebright, Y.W., and Ebright, R.H. 1994. Identification of the target of a transcription activator protein by proteinprotein photocrosslinking. Science 265: 90-92.

Cheng, J.X., Floer, M., Ononaji, P., Bryant, G., and Ptashne, M. 2002. Responses of four yeast genes to changes in the transcriptional machinery are determined by their promoters. Curr. Biol. 12: 1828-1832.

Chi, T., Lieberman, P., Ellwood, K., and Carey, M. 1995. A general mechanism for transcriptional synergy by eukaryotic activators. Nature 377: 254-257.

Cismowski, M.J., Laff, G.M., Solomon, M.J., and Reed, S.I. 1995. KIN28 encodes a C-terminal domain kinase that controls mRNA transcription in S. cerevisiae but lacks cyclin-dependent kinase-activating kinase (CAK) activity. Mol. Cell. Biol. 15: 2983-2992.

Cosma, M.P. 2002. Ordered recruitment: Gene-specific mechanism of transcription activation. Mol. Cell 10: 227-236.

Datwyler, S.A. and Meares, C.F. 2000. Protein-protein interactions mapped by artificial proteases: Where $\sigma$ factors bind to RNA polymerase. Trends Biochem. Sci. 25: 408-414.
Dudley, A.M., Rougeulle, C., and Winston, F. 1999. The Spt components of SAGA facilitate TBP binding to a promoter at a post-activator-binding step in vivo. Genes \& Dev. 13: 2940-2945.

Eisenmann, D.M., Arndt, K.M., Ricupero, S.L., Rooney, J.W., and Winston, F. 1992. SPT3 interacts with TFIID to allow normal transcription in S. cerevisiae. Genes \& Dev. 6: 13191331.

Eisenmann, D.M., Chapon, C., Roberts, S.M., Dollard, C., and Winston, F. 1994. The Saccharomyces cerevisiae SPT8 gene encodes a very acidic protein that is functionally related to SPT3 and TATA-binding protein. Genetics 137: 647-657.

Geiger, J.H., Hahn, S., Lee, S., and Sigler, P.B. 1996. Crystal structure of the yeast TFIIA/TBP/DNA complex. Science 272: 830-836.

Grant, P.A., Schieltz, D., Pray-Grant, M.G., Steger, D.J., Reese, J.C., Yates III, J.R., and Workman, J.L. 1998. A subset of Taf(II)s are integral components of the SAGA complex required for nucleosome acetylation and transcriptional stimulation. Cell 94: 45-53.

Hampsey, M. 1998. Molecular genetics of the RNA polymerase II general transcriptional machinery. Microbiol. Mol. Biol. Rev. 62: 465-503.

Kang, J.J., Auble, D.T., Ranish, J.A., and Hahn, S. 1995. Analysis of the yeast transcription factor TFIIA: Distinct functional regions and a polymerase II-specific role in basal and activated transcription. Mol. Cell. Biol. 15: 1234-1243.

Kobayashi, N., Horn, P.J., Sullivan, S.M., Triezenberg, S.J., Boyer, T.G., and Berk, A.J. 1998. DA-complex assembly activity required for VP16C transcriptional activation. Mol. Cell. Biol. 18: 4023-4031.

Kokubo, T., Swanson, M.J., Nishikawa, J.I., Hinnebusch, A.G., and Nakatani, Y. 1998. The yeast Taf145 inhibitory domain and TFIIA competitively bind to TATA-binding protein. Mol. Cell. Biol. 18: 1003-1012.

Kraemer, S.M., Ranallo, R.T., Ogg, R.C., and Stargell, L.A. 2001. TFIIA interacts with TFIID via association with TATA-binding protein and Taf40. Mol. Cell. Biol. 21: 1737-1746.

Kuras, L. and Struhl, K. 1999. Binding of TBP to promoters in vivo is stimulated by activators and requires Pol II holoenzyme. Nature 399: 609-613.

Larschan, E. and Winston, F. 2001. The S. cerevisiae SAGA complex functions in vivo as a coactivator for transcriptional activation by Gal4. Genes \& Dev. 15: 1946-1956.

Lee, T.I., Causton, H.C., Holstege, F.C., Shen, W.C., Hannett, N., Jennings, E.G., Winston, F., Green, M.R., and Young, R.A. 2000. Redundant roles for the TFIID and SAGA complexes in global transcription. Nature 405: 701-704.

Li, X.Y., Virbasius, A., Zhu, X., and Green, M.R. 1999. Enhancement of TBP binding by activators and general transcription factors. Nature 399: 605-609.

Lieberman, P.M. and Berk, A.J. 1994. A mechanism for Tafs in transcriptional activation: Activation domain enhancement of TFIID-TFIIA-promoter DNA complex formation. Genes \& Dev. 9: 995-1006.

Liu, Q., Gabriel, S.E., Roinick, K.L., Ward, R.D., and Arndt, K.M. 1999. Analysis of TFIIA function in vivo: Evidence for a role in TATA-binding protein recruitment and gene-specific activation. Mol. Cell. Biol. 19: 8673-8685.

Liu, Y., Kung, C., Fishburn, J., Ansari, A.Z., Shokat, K.M., and Hahn, S. 2004. Two cyclin-dependent kinases promote RNA polymerase II transcription and formation of the Scaffold Complex. Mol. Cell. Biol. 24: 1721-1735.

Madison, J.M. and Winston, F. 1997. Evidence that Spt3 functionally interacts with Mot1, TFIIA, and TBP to confer promoter-specific transcriptional control in S. cerevisiae. Mol. 
Cell. Biol. 17: 287-295.

Mencia, M., Moqtaderi, Z., Geisberg, J.V., Kuras, L., and Struhl, K. 2002. Activator-specific recruitment of TFIID and regulation of ribosomal protein genes in yeast. Mol. Cell 9: 823833.

Orphanides, G., Lagrange, T., and Reinberg, D. 1996. The general transcription factors of RNA polymerase II. Genes \& Dev. 10: 2657-2683.

Ozer, J., Moore, P.A., Bolden, A.H., Lee, A., Rosen, C.A., and Lieberman, P.M. 1994. Molecular cloning of the small (g) subunit of human TFIIA reveals functions critical for activated transcription. Genes \& Dev. 8: 2324-2335.

Ozer, J., Lezina, L.E., Ewing, J., Audi, S., and Lieberman, P.M. 1998a. Association of transcription factor IIA with TBP is required for transcriptional activation of a subset of promoters and cell cycle progression in S. cerevisiae. Mol. Cell. Biol. 18: 2559-2570.

Ozer, J., Mitsouras, K., Zerby, D., Carey, M., and Lieberman, P.M. 1998b. Transcription factor IIA derepresses TATAbinding protein (TBP)-associated factor inhibition of TBPDNA binding. J. Biol. Chem. 273: 14293-14300.

Pereira, L.A., Klejman, M.P., and Timmers, H.T. 2003. Roles for BTaf 1 and Motlp in dynamics of TATA-binding protein and regulation of RNA polymerase II transcription. Gene 315: 113.

Pray-Grant, M.G., Schieltz, D., McMahon, S.J., Wood, J.M., Kennedy, E.L., Cook, R.G., Workman, J.L., Yates III, J.L., and Grant, P.A. 2002. The novel SLIK histone acetyltransferase complex functions in the yeast retrograde response pathway. Mol. Cell. Biol. 22: 8774-8786.

Ranish, J.A., Yudkovsky, N., and Hahn, S. 1999. Intermediates in formation and activity of the RNA polymerase II preinitiation complex: Holoenzyme recruitment and a postrecruitment role for the TATA box and TFIIB. Genes \& Dev. 13: 49-63.

Reddy, P. and Hahn, S. 1991. Dominant negative mutations in yeast TFIID define a bipartite DNA-binding region. Cell 65: 349-357.

Saleh, A., Lang, V., Cook, R., and Brandl, C.J. 1997. Identification of native complexes containing the yeast coactivator/ repressor proteins NGG1/ADA3 and ADA2. J. Biol. Chem. 272: 5571-5578.

Sanders, S.L., Jennings, J., Canutescu, A., Link, A.J., and Weil, P.A. 2002. Proteomics of the eukaryotic transcription machinery: Identification of proteins associated with components of yeast TFIID by multidimensional mass spectrometry. Mol. Cell. Biol. 22: 4723-4738.

Shen, W.-C. and Green, M.R. 1997. Yeast TafII145 functions as a core promoter selectivity factor, not a general coactivator. Cell 90: 615-624.

Stargell, L.A., Moqtaderi, Z., Dorris, D.R., Ogg, R.C., and Struhl, K. 2000. TFIIA has activator-dependent and core promoter functions in vivo. J. Biol. Chem. 275: 12374-12380.

Sterner, D.E., Grant, P.A., Roberts, S.M., Duggan, L.J., Belotserkovskaya, R., Pacella, L.A., Winston, F., Workman, J.L., and Berger, S.L. 1999. Functional organization of the yeast SAGA complex: Distinct components involved in structural integrity, nucleosome acetylation, and TATA-binding protein interaction. Mol. Cell. Biol. 19: 86-98.

Sterner, D.E., Belotserkovskaya, R., and Berger, S.L. 2002. SALSA, a variant of yeast SAGA, contains truncated Spt7, which correlates with activated transcription. Proc. Natl. Acad. Sci. 99: 11622-11627.

Tan, S., Hunziker, Y., Sargent, D.F., and Richmond, T.J. 1996. Crystal structure of a yeast TFIIA/TBP/DNA complex. $\mathrm{Na}$ ture 381: 127-134.
Wang, W., Gralla, J.D., and Carey, M. 1992. The acidic activator GAL4-AH can stimulate polymerase II transcription by promoting assembly of a closed complex requiring TFIID and TFIIA. Genes \& Dev. 6: 1716-1727.

Weideman, C.A., Netter, R.C., Benjamin, L.R., McAllister, J.J., Schmiedekamp, L.A., Coleman, R.A., and Pugh, B.F. 1997. Dynamic interplay of TFIIA, TBP, and TATA DNA. J. Mol. Biol. 271: 61-75.

Wu, P.Y. and Winston, F. 2002. Analysis of Spt7 function in the Saccharomyces cerevisiae SAGA coactivator complex. Mol. Cell. Biol. 22: 5367-5379.

Yokomori, K., Admon, A., Goodrich, J.A., Chen, J.L., and Tjian, R. 1993. Drosophila TFIIA-L is processed into two subunits that are associated with the TBP/Taf complex. Genes \& Dev. 7: 2235-2245.

Yudkovsky, N. 2001. "Mechanisms of factor recruitment at promoters during RNA polymerase II transcription." Ph.D. thesis, University of Washington and Fred Hutchinson Cancer Center, Seattle.

Yudkovsky, N., Ranish, J.A., and Hahn, S. 2000. A transcription reinitiation intermediate that is stabilized by activator. $\mathrm{Na}$ ture 408: 225-229. 


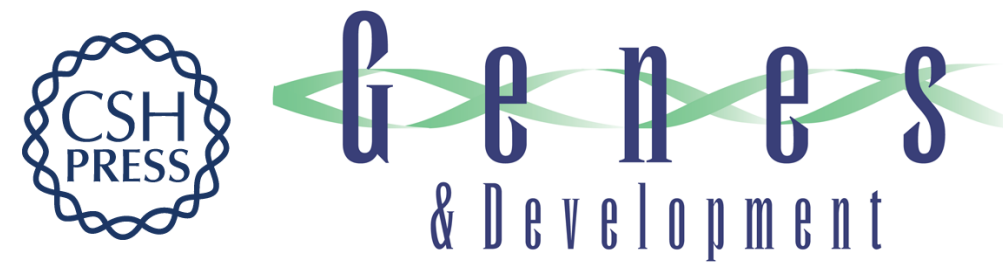

\section{Positive and negative functions of the SAGA complex mediated through interaction of Spt8 with TBP and the N-terminal domain of TFIIA}

Linda Warfield, Jeffrey A. Ranish and Steven Hahn

Genes Dev. 2004, 18:

Access the most recent version at doi:10.1101/gad.1192204

Supplemental http://genesdev.cshlp.org/content/suppl/2004/05/07/18.9.1022.DC1

Material

References This article cites 53 articles, 33 of which can be accessed free at: http://genesdev.cshlp.org/content/18/9/1022.full.html\#ref-list-1

License

Email Alerting Receive free email alerts when new articles cite this article - sign up in the box at the top Service right corner of the article or click here.

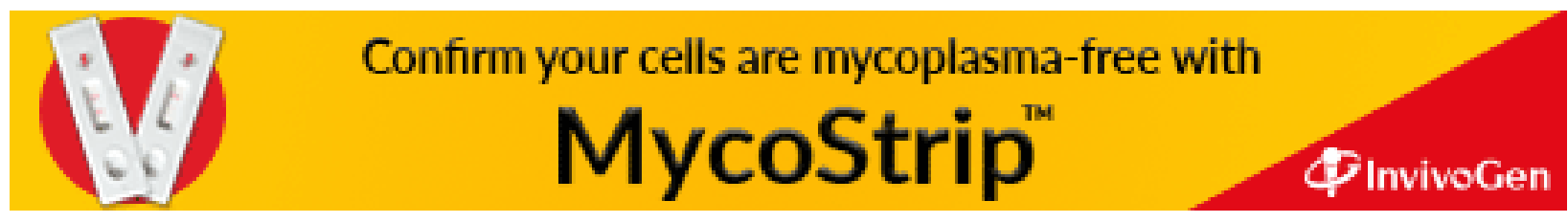

